(RESEARCH ARTICLE)

\title{
Metabolic blocks and insulin resistance
}

\author{
Emil Mukhamejanov *, Nailya Ibragimova and Arailym Aitynova
}

JSC "Scientific Center of Anti-infectious Drugs", Kazakhstan, Almaty.

Publication history: Received on 03 May 2020; revised on 09 May 2020; accepted on 11 May 2020

Article DOI: https://doi.org/10.30574/wjarr.2020.6.2.0140

\begin{abstract}
Violation of glucose intake and oxidation in muscle tissue can occur at various stages and, according to the feedback principle, this will lead to inhibition of glucose utilization or the development of insulin resistance. In this regard, the process of violation of an excess glucose stream deposition in the form of glycogen (glycogen block) is considered. The processes of violation of glucose oxidation at various stages are considered: 1) pyruvate conversion (pyruvate block); 2) excessive accumulation of lactate (lactate block); 3) deterioration of the process of transamination of pyruvate (BCAA block); 4) impaired transport of reduced equivalents to mitochondria (aspartate block); 5) deficiency of cofactors for the conversion of pyruvate to acetyl-CoA and oxaloacetate; 6) carnitine deficiency for the transfer of acetyl-CoA to mitochondria (carnitine block); 7) a decrease in the rate of acetyl CoA entry into mitochondria due to a decrease in their number (mitochondrial block); 8) a decrease in the level of coenzyme Q10 and a decrease in the rate of oxidative phosphorylation (oxidative block). These disorders (metabolic blocks) can be corrected by providing substrate for these reactions, which may be the principle for the prevention and treatment of insulin-resistant conditions.
\end{abstract}

Keywords: Insulin resistance; Metabolic blocks; Diabetes; Prevention; Treatment

\section{Introduction}

Diabetes mellitus (DM) is one of the most common metabolic diseases worldwide, and the number of patients with DM has continued to increase in recent years. Patients with DM exhibit hyperglycemia caused by an impairment in insulin secretion (type 1), insulin action (type 2), or both. Type 1 diabetes mellitus (T1DM), which accounts for less than $10 \%$ of diabetes cases, is characterized by an immune-mediated destruction of $\beta$ cells in the pancreatic islets of Langerhans, leading to insulin deficiency [1]. On the other hand, type 2 diabetes mellitus (T2DM), which accounts for less than $90 \%$ of diabetes cases, involves insulin resistance in peripheral tissues and increased levels of blood glucose due to overnutrition accompanied by deficient insulin secretion [2]. DM is often associated with the development of secondary complications in various organs, such as eyes, kidneys, heart, brain, and skeletal muscle [3].

\section{Material and methods}

The article used PubMed data and the results of our own theoretical developments regarding the metabolic blocks for individual stages of glucose utilization.

\section{Results and discussion}

While infectious agents were the major causes of disease at the beginning of the 20th century, infectious diseases were replaced by type 2 diabetes mellitus (T2DM) and diabetic complications, cardiovascular diseases (CVD) and cancer as major causes of death by the 21st century. At present, westernized populations are plagued by a plethora of chronic degenerative diseases, including obesity, T2DM, atherosclerosis, coronary heart disease, stroke, autoimmune diseases,

\footnotetext{
${ }^{*}$ Corresponding author: Emil Mukhamejanov
} 
essential hypertension, cancer, osteoporosis and other more, and the number of these diseases is also rapidly increasing in developing countries [4].

Since the main negative manifestation of diabetes is hyperglycemia, all technologies for the prevention and treatment of diabetes are aimed to reduce amount of carbohydrate intake and increase amount spent on physical activity [5]. This principle has been supported since the last century, but the number of patients with diabetes is increasing from year to year and the disease has become epidemic of non-communicable origin. It should be noted that a person is a weak creature and wants to eat and lie on the couch, so the bulk of the population does not listen to medicinal advice, so the world is still losing the fight against diabetes.

It is currently believed that impaired glucose homeostasis is associated with the development of insulin resistance (IR) [6], which is the main step in the pathogenesis of diabetes and development of side effects of this disease.

Oxidation of glucose is a multi-stage process and at its stages problems with its course may appear. A decrease in the rate of glucose oxidation at any stage automatically leads to inhibition of all previous reactions (metabolic blocks), since the reaction product inhibits the reaction itself and, ultimately, leads to inhibition of glucose into the cell, therefore, in this article we will try to understand the reasons of impaired glucose oxidation and ways to prevent them.

Carbohydrates make up half of the diet calorie content and for an ordinary person with a need of $3000 \mathrm{kcal}$ they make up $375 \mathrm{~g}$. In the blood (with a norm of blood sugar of $100 \mathrm{mg} \%$ and a total blood volume of $5 \mathrm{l}$ ), only $5 \mathrm{~g}$ of glucose is contained. In the liver in the form of glycogen contains about $70 \mathrm{~g}$, and in the muscles about $300 \mathrm{~g}[7,8]$, i.e. in total, this also amounts to $375 \mathrm{~g}$ or there is a correspondence (homeostasis) between intake and carbohydrate content.

It can be seen from the above data that $80 \%$ of glucose stores are in the muscles, which is consistent with the data that the muscles determine $80 \%$ of glucose uptake stimulated by insulin, $[9,10]$, therefore, in the development mechanism of IR, we must first consider why metabolic blocks of glucose oxidation occur skeletal muscle.

The skeletal muscle is notably affected by DM. It has been shown that DM induces atrophy [11], fiber-type transition from oxidative to glycolytic [12], and impaired energy metabolism in skeletal muscle [13]. These alterations result in skeletal muscle dysfunction, such as muscle weakness and exercise intolerance [14].

There is no pure glucose in the muscle cell, since when it is received from the blood, it undergoes phosphorylation (ATP energy is expended) with the participation of the hexokinase enzyme and glucose-6-phosphate (G-6-F) is formed. This, on the one hand, prevents the return of glucose from the cell, since the enzyme glucose-6-phosphatase is absent in the muscles, and, on the other hand, activated glucose undergoes further conversion more easily.

The amount of glycogen in the muscles does not exceed $1 \%$, since glycogen has a branching structure and attracts a lot of water due to hydrophilicity, therefore it takes up a lot of space in the cell. In connection with a change in the lifestyle of a modern person, a decrease in physical activity has occurred, which leads to a decrease in glycogen utilization. Therefore, the possibility of turning G-6-F into glycogen is reduced, since the place simply turns out to be occupied. This is the so-called "glycogen block", therefore, programs to increase physical activity, which are aimed at removing the glycogen block, are quite legitimate.

In repeated phosphorylation with the participation of the enzyme phosphofructokinase, conditions are created for the reaction of decomposition of hexose to triosis and the subsequent formation of pyruvic acid (pyruvate). In this case, formation of 4 ATP molecules occurs. If we take into account that at first 2 ATP molecules were expended, then the ATP yield during glycolysis is 2 ATP molecules per glucose molecule.

In patients with diabetes, the absorption of glucose by the muscles decreases under the influence of insulin $[15,16]$, therefore, it is believed that insulin should activate the key glycolysis enzymes, but insulin does not affect the activity of hexokinase and phosphofructokinase, and only the allosteric principle is detected [17].

Pyruvate formed during glycolysis can be converted in several directions, and all compounds lying at the crossroads of metabolic pathways should be maintained at the homeostatic level. Therefore, in case of violation of the further conversion of pyruvate, its formation is automatically inhibited or glucose uptake is stopped. This is the so-called "pyruvate block". Indeed, violation of pyruvate metabolism is the reason for development of a number of metabolic diseases $[18,19]$. 
During glucose oxidation or its dehydrogenation, reduced equivalents are released in the form of NAD.H2. However, the accumulation of NAD.H2 leads to disruption of redox reactions activity, which is a threat for vital processes and the task of its fraternal oxidation to NAD arises. NAD.H2 oxidation occurs in the mitochondria, but the mitochondrial membrane is not permeable to NAD.H2. The malate/aspartate shunt acts as the carrier of the reduced equivalents, the limiting link in which is the amino acid asparticity (aspartate), therefore, with aspartate deficiency, the possibility of NAD.H2 transfer to mitochondria decreases and the so-called aspartate block develops. Indeed, in sports practice, aspartate feed is used as an ergogenic compound to increase physical performance [20].

Under anaerobic conditions, hydrogen (H2) can join pyruvate and lactic acid (lactate) is formed. However, accumulation of lactate leads to the development of lactic acidosis and impaired contractile function of muscle fiber. In addition, developing acidosis can cause development of oxidative stress, which negatively affects the muscle fiber vital activity, so the cell must protect itself from excessive lactate formation by reducing glucose intake or developing IR [21], therefore, development of "lactate block" and IR in this case should be considered as an adaptive reaction of the body to prevent cell death. The cell is forced to free itself from lactate by transporting it into the circulation. Further, lactate enters the liver, in which it serves as a substrate for gluconeogenesis, but insulin inhibits gluconeogenesis and activates the process of converting lactate into lipids, which contributes to the development of lipidemia and increased fat deposition in adipocytes, therefore, one of the markers of obesity is an increase in blood lactate [22].

For reduction of lactate formation, it is advisable to convert pyruvate to the amino acid alanine, but this requires donors of amino groups, which are branch chain amino acids (BCAAs), therefore, when they are deficient, the possibility of converting pyruvate to alanine decreases and the manifestations of the BCAA block develop, which contributes to the development of pyruvate block. Therefore, when taking BCAAs, glucose utilization increases [23,24].

For conversion of pyruvate under aerobic conditions, carbon must be removed from it with participation of the pyruvate dehydrogenase complex or, conversely, carbon should be attached with the participation of pyruvate carboxylase. At the same time, acetyl-CoA and oxaloacetate are formed, but the activity of these enzymes requires cofactors, which are vitamin B1, magnesium ions and lipoic acid, so when they are deficient, [25,26,27] conversion of pyruvate to acetyl-CoA and oxaloacetate decreases and manifestations of the pyruvate block develop IR.

Acetyl-CoA is formed in the cytoplasm, and its oxidation occurs in the mitochondria, but the mitochondrial membrane is not permeable to acetyl-CoA, therefore, a carrier, which is carnitine, is required, therefore, if it is deficient, acetyl-CoA transfer is disrupted, which leads to a feedback principle of inhibition of its formation or a violation of the pyruvate conversion, and a pyruvate block develops. Consumption of carnitine improves acetyl-CoA transport and oxidation [28]. This is the so-called "carnitine block".

Acetyl-CoA also can not enter the mitochondria with a decrease in their number [29], functional impairment [30], in particular under the influence of excess free radical production [31]. This is the so-called mitochondrial block. In this case, according to the feedback principle, the conversion of pyruvate to acetyl-CoA is inhibited and the manifestations of the pyruvate block develop.

Upon entry into mitochondria, acetyl-CoA dehydrogenation occurs and reduced equivalents are formed, which enter the biological oxidation chain. At the first stage of biological oxidation, ubiquinone or coenzyme $\mathrm{Q}_{10}$. (CoQ $\left.{ }_{10}\right)$ appears. However, $\mathrm{CoQ}_{10}$ decreases with age [32], which leads to a decrease in the value of oxidative phosphorylation and acetylCoA dehydrogenation and its entry into mitochondria are inhibited by the feedback principle. This is the so-called "oxidative block". When taking $\mathrm{CoQ}_{10}$, the oxidative phosphorylation process improves and the manifestations of IR are reduced. [33].

In case of impaired glucose metabolism at various stages of its oxidation, the mechanism of maintaining homeostasis is activated by transferring accumulating metabolites by including alternative pathways for their metabolism. Since the possibilities of G-6-F deposition in the form of glycogen are limited, the conversion of G-6-F to fructose and sorbitol is enhanced, the accumulation of poorly digestible carbohydrates in the cell leads to the formation of conglomerates and impaired cell function, therefore, the cell protection mechanism in the form of the development of IR and a decrease in the intake of glucose in the muscles. Another alternative way is to convert under-oxidized metabolic products with excessive secretion of insulin into fats. In metabolic terms, this pathway is shown in Figure 1. 


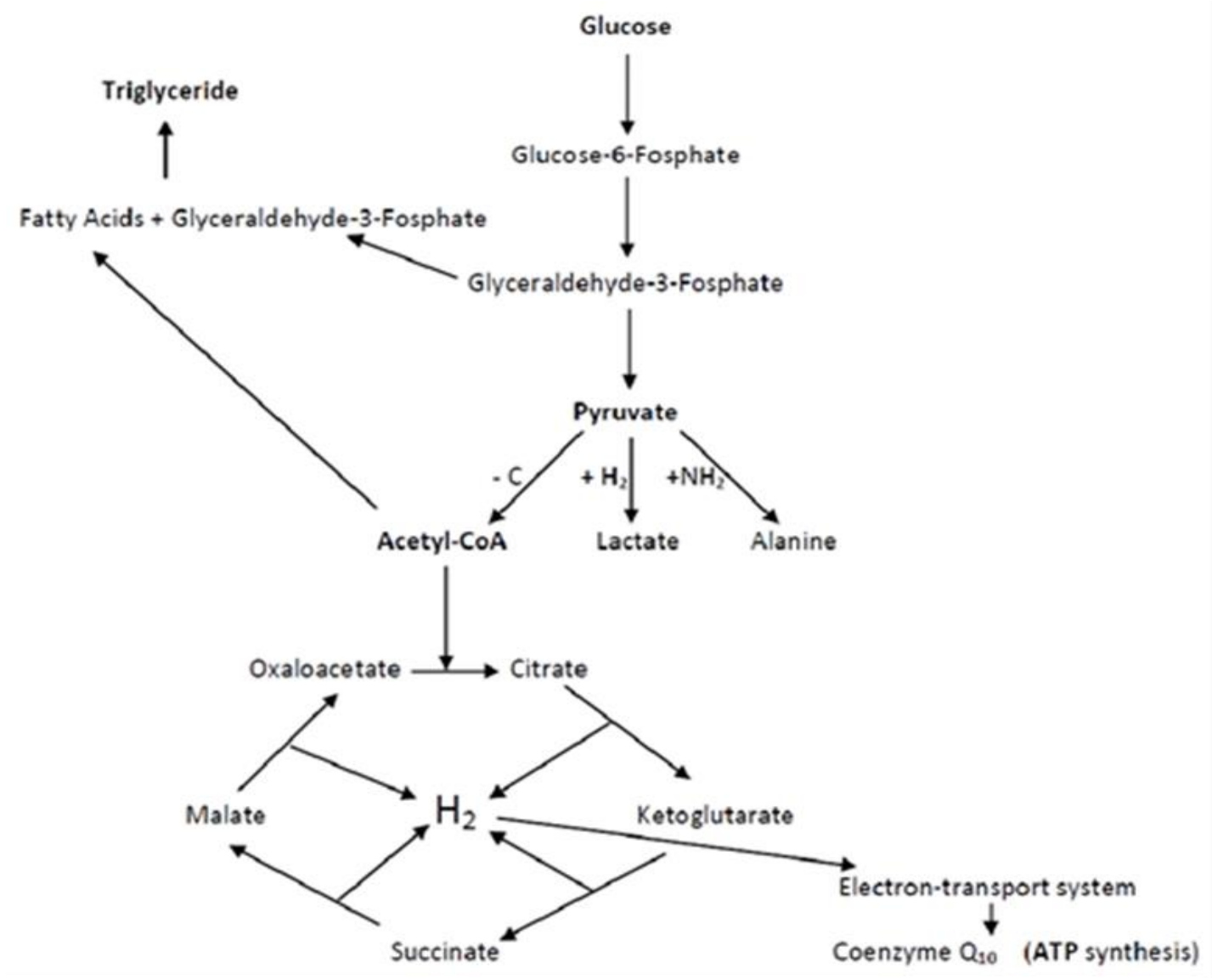

Figure 1 Diagram of glucose oxidation and lipogenesis activation pathway in case of impaired conversion of glyceraldehyde-3-phosphate and acetyl-CoA

As can be seen from Figure 1, if glucose oxidation worsens at the stages of glyceraldehyde-3-phosphate and acetyl-CoA oxidation, their carbon skeleton is discharged into fats or lipogenesis is activated. These manifestations are noted when trying to increase the flow of glucose into the muscles under the influence of pharmaceuticals. Therefore, it is more advisable to improve the course of glucose oxidation, rather than just trying to increase its uptake into tissues with the help of pharmaceuticals, since in this case we can get negative manifestations in the form of activation of lipogenesis. The accumulation of lipids in the muscles can also have a negative effect on their function and, accordingly, prevent the negative effects of lipid accumulation, glucose intake decreases due to the development of IR [34]. This manifestation can be called a "lipid block".

ATP is formed in the biological oxidation chain, but the ATP level in the muscle cell is at a constant level even during the feeding / starvation cycle or during exercise. Therefore, the ATP/ADP coefficient is an information signal for regulatory systems in relation to maintaining ATP homeostasis. With a decrease in the use of ATP, an increase in the coefficient and inhibition of hexokinase, i.e. muscle glucose is inhibited and IR develops. This is a kind of "energy block". With an increase in ATP utilization, the ATP/ADP coefficient decreases and hexokinase is activated, which contributes to an increase in the supply of glucose to the muscles and decreases the manifestations of IR.

The main consumers of ATP in muscles are physical activity and protein synthesis. There are many serious works on the role of physical activity in the regulation of glucose homeostasis, but virtually no attention is paid to the role of protein synthesis in the regulation of glucose homeostasis. It is known that 3 ATP is expended on the formation of a peptide bond. The average protein contains 100 peptide bonds. Thousands of proteins are synthesized per day, so protein synthesis is the most energy-intensive process in a muscle cell.

Insulin is an anabolic hormone; therefore, it is involved only in the processes of storage of glucose either in the form of glycogen or in the form of lipids. But it does not show catabolic properties or does not affect the process of glucose catabolism, therefore, insulin should be considered as a marker of "excess" glucose and amino acids. 
Protein synthesis occurs on ribosomes even under fasting conditions (energy deficiency) to preserve the synthesis of important functional proteins. With an excess of glucose and amino acids after eating (excess energy), insulin secretion is enhanced and anabolic processes are activated.

The effect of insulin on protein metabolism is associated with its ability to initiate aggregation of ribosomes into polysomes or its effect on protein metabolism is manifested at the stage of translation [35]. Translation is the fastest process for regulating the rate of protein synthesis (Fig. 2).

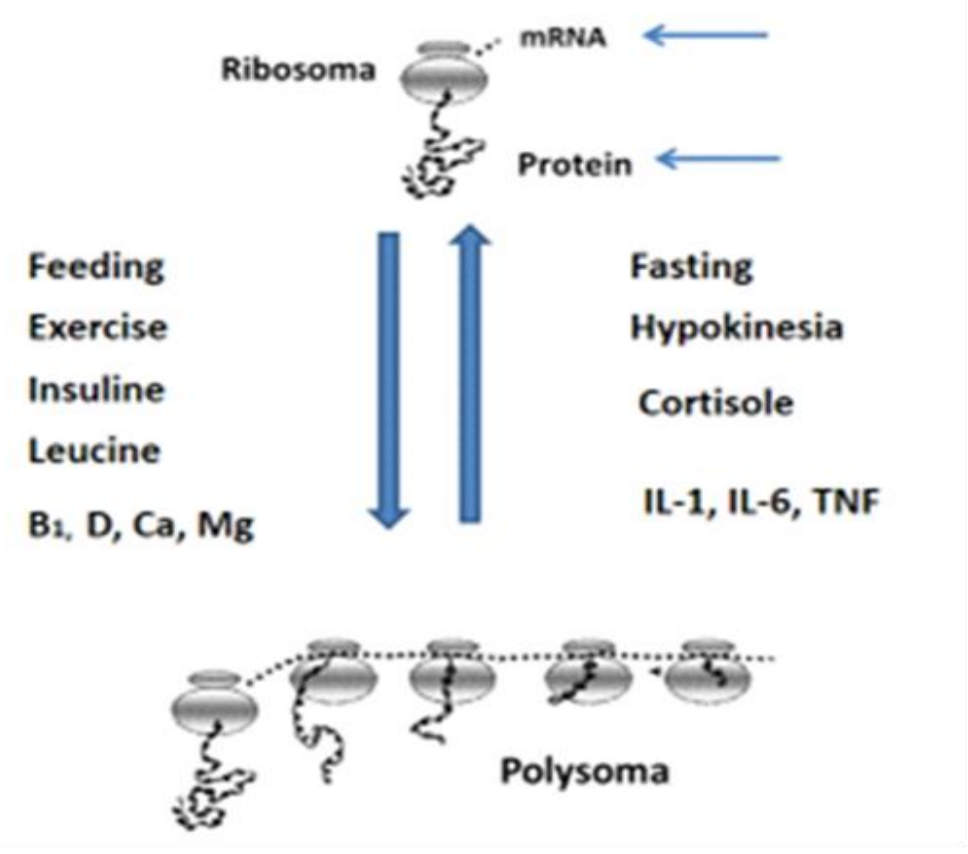

Figure 2 Factors affecting the regulation of protein synthesis at the translation stages

As can be seen from Figure 2, a number of factors affect the rate of protein synthesis. This is feeding, the hormone insulin, and physical activity, especially of a resistant nature [36]. Some vitamins have a positive effect on protein synthesis [37,38] and microelements [39]. This effect is achieved through the activation of mTOR kinase or translation kinase $[40,41]$, which promotes aggregation of ribosomes into polysomes. In this case, the rate of protein synthesis increases by about 10 times. With an increase in the rate of protein synthesis, the ATP energy requirements automatically increase and, accordingly, glucose utilization increases and the manifestations of IR are removed.

It should be noted that under the influence of insulin the process of lipolysis and fat oxidation is inhibited, therefore lipids cannot serve as ATP energy sources for protein synthesis during food intake and ATP formed due to protein synthesis during this period can be used glucose catabolism. Therefore, when lowering blood glucose levels, insulin levels decrease and anabolic processes are inhibited, and all hypoglycemic states are only catabolic.

Polysomes can decay back to ribosomes. This occurs during fasting (deficiency of the substrate for protein synthesis), during hypokinesia (reducing the anabolic effect of physical activity), under the action of cortisol (hormone stress), and under the influence of inflammatory cytokines (switching the flow of energy to inflammatory processes). In all these cases, amount of protein synthesis decreases and, accordingly, need of glucose energy for anabolic process, therefore, under these conditions, amount of glucose utilization by the muscles decreases or the manifestations of IR develop $[42,43]$.

Usually, muscles are considered as an organ providing mobility of the body, but muscles take an important role in the regulation of metabolic processes, in particular in glucose homeostasis. With insufficient glucose intake, for example, during fasting, homeostasis is maintained due to endogenous synthesis (gluconeogenesis), but proteins are used as a substrate.

Since muscles make up about $50 \%$ of the protein mass, respectively, they mainly provide the supply of the substrate for the gluconeogenesis process. Therefore, with a decrease in muscle mass - sarcopenia (from the Greek words sacra meat and singing - deficiency), the amount of glucose utilization decreases, which is the main reason for the increase in 
its concentration in the blood (glycemia) and there is an increase in insulin secretion (insulinemia). This is an important reason for the development of T2DM. Under the influence of insulin, the discharge of the carbon skeleton of glucose into fats is enhanced, which leads to the development of dyslipidemia. This contributes to increased fat deposition in adipocytes and the development of obesity.

During sarcopenia possibility of supplying a substrate (amino acids) for gluconeogenesis is reduced. These manifestations are especially dangerous during the period of nightly starvation, when the risk of developing deep hypoglycemia and even death develops. Therefore, maintenance of glucose homeostasis during feeding and fasting significantly worsens during sarcopenia, and in these cases it is necessary to increase attention to the development of measures to increase muscle mass.

Thus, diabetes is a violation of protein metabolism, so all measures to improve the process of protein synthesis will increase the need for ATP and indirectly increase the flow of glucose into the muscle cell to provide the anabolic process with energy. This requires a review of our understanding of the pathogenesis of diabetes and allows us to develop new technologies for the prevention and treatment of diabetes. These provisions apply to type 1 diabetes, when an improvement in protein synthesis can help reduce glycemia even without increasing the dose of insulin.

\section{Conclusion}

In the mechanism of insulin resistance development, deterioration of glucose oxidation during its anaerobic and aerobic conversion plays a significant role. These disorders or metabolic blocks can be eliminated by using of food compounds, which must be taken into account when developing preventive and therapeutic measures.

\section{Compliance with ethical standards}

\section{Disclosure of conflict of interest}

Disclosure of conflict of interest Conflicts Authors declare that the research was conducted in the absence of any commercial or financial relationships that could be construed as a potential conflict of interest.

\section{References}

[1] American Diabetes Association Diagnosis and classification of diabetes mellitus. (2011). Diabetes Care, 34, 6269.

[2] Groop LC and Eriksson JG. (1992). The etiology and pathogenesis of non-insulin-dependent diabetes Ann. Med, 24, 483-489.

[3] Gispen WH and Biessels GJ. (2000) Cognition and synaptic plasticity in diabetes mellitus. Trends Neurosci, 23, 542-549.

[4] Fung J and Berger A. (2016). Hyperinsulinemia and insulin resistance: scope of the problem. J Insulin Resist, 1, 16.

[5] Trnovec T, Cook T, Kahayová K and Nyulassy S. (2001). Civilization as a threat to human health? Cent Eur J Public Health, 9, 49-52.

[6] Ryan DH. (2003). Diet and exercise in the prevention of diabetes. Int. J. Clin. Pract. Suppl, 134, 28-35.

[7] Schwarz PEH, Gruhl U, Schuppenies A, Schulze J and Bornstein SR. (2007). Prevention of Diabetes Mellitus. The Future of German Diabetology Hamostaseologie, 27, 13-21.

[8] Koffler M, Ramirez LC and Raskin P. (1989). Insulin Resistance and Diabetes, Mechanism and Possible Intervention Diabetes. Res Clin Pract, 7, 83-98.

[9] Nilsson LH and Hultman E. (1974). Liver and Muscle Glycogen in Man after Glucose and Fructose Infusion. Scand J. Clin. Lab. Invest, 33, 5-10.

[10] Macauley M, Smith FE, Thelwall PE, Hollingsworth KG and Taylor R. (2015). Diurnal Variation in Skeletal Muscle and Liver Glycogen in Humans with Normal Health and Type 2 Diabetes Clin. Sci. (Lond), 128, 707-713.

[11] Deshmukh AS. (2016). Insulin-stimulated Glucose Uptake in Healthy and Insulin-Resistant Skeletal Muscle. Horm. Mol. Biol. Clin. Investig, 26, 13-24. 
[12] Deshmukh AS. (2016). Proteomics of Skeletal Muscle: Focus on Insulin Resistance and Exercise. Biology Proteomes, 4,1 .

[13] Andersen H, Gadeberg PC, Brock B and Jakobsen J. (1997). Muscular atrophy in diabetic neuropathy: A stereological magnetic resonance imaging study. Diabetologia, 40, 1062-1069.

[14] Oberbach A, Bossenz Y, Lehmann S, Niebauer J, Adams V, Paschke R, Schon MR, Bluher M and Punkt K. (2006). Altered fiber distribution and fiber-specific glycolytic and oxidative enzyme activity in skeletal muscle of patients with type 2 diabetes. Diabetes Care, 29, 895-900.

[15] Brandenburg SL, Reusch JE, Bauer TA, Jeffers BW, Hiatt WR and Regensteiner JG. (1999). Effects of exercise training on oxygen uptake kinetic responses in women with type 2 diabetes. Diabetes Care, 22, 1640-1646.

[16] Regensteiner JG, Sippel J, McFarling ET, Wolfel EE and Hiatt WR. (1995). Effects of non-insulin-dependent diabetes on oxygen consumption during treadmill exercise. Med. Sci. Sports Exerc, 27, 875-881.

[17] Kelley DE, Mintun MA, Watkins SC, Simoneau JA, Jadali F, Fredrickson A, Beattie J and Theriault R. (1996). The effect of non-insulin-dependent diabetes mellitus and obesity on glucose transport and phosphorylation in skeletal muscle. J.Clin.Invest, 97, 2705-2713.

[18] Hickey MS, Carey JO, Azevedo JL, Houmard JA, Pories WJ, Israel RG and Dohm GL. (1995) Skeletal muscle fiber composition is related to adiposity and in vitro glucose transport rate in humans. Am.J.Physiol, 268, 453-457.

[19] Vestergaard H. (1999). Studies of gene expression and activity of hexokinase, phosphofructokinase and glycogen synthase in human skeletal muscle in states of altered insulin-stimulated glucose metabolism. Dan.Med.Bull, 46, 13-34.

[20] Gray LR, Tompkins SC and Taylor EB. (2014). Regulation of Pyruvate Metabolism and Human Disease. Cell Mol Life Sci, 71, 2577-604.

[21] Vanderperre B, Bender T, Kunji ERS and Martinou J-C. (2015). Mitochondrial Pyruvate Import and Its Effects on Homeostasis. Curr Opin Cell Biol, 33, 35-41.

[22] Trudeau F. (2008). Aspartate as an ergogenic supplement. Sports Med, 38, 9-16.

[23] Williams RS, Heilbronn LK, Chen DL, Coster ACF, Greenfield JR and Samocha-Bonet D. (2016). Dietary Acid Load, Metabolic Acidosis and Insulin Resistance - Lessons From Cross-Sectional and Overfeeding Studies in Humans. Clin Nutr, 35, 1084-90.

[24] Adeva-Andany M, Lopez-Ojen M, Funcasta-Caldeon R, Ameneiros-Rodriguez E, Donapetry-Garcia C, Vila-Altesor M and Rodriguez-Seijas J. (2014). Comprehensive Review on Lactate Metabolism in Human Health. Mitochondrion, 17, 76-100.

[25] Doi M, Yamaoka I, Nakayama M, Mochizuki S, Sugahara K and Yoshizawa F. (2005). Isoleucine, a blood glucoselowering amino acid, increases glucose uptake in rat skeletal muscle in the absence of increases in AMP-activated protein kinase activity. J.Nutr., 135, 2103-8.

[26] Blomstrand E, Eliasson J, Karlsson HKR and Kohnke R. (2006). Branched-chain amino acids activate key enzymes in protein synthesis after physical exercise. J Nutr, 136, 269-273.

[27] Habeb AM, Flanagan SE and Zulali MA. (2018). Pharmacogenomics in diabetes outcomes of thiamine therapy in TRMA syndrome. Diabetologia, 61, 1027-1036.

[28] Ramadass S, Basu S and Srinivasan AR. (2015). SERUM magnesium levels as an indicator of status of Diabetes Mellitus type 2. Diabetes Metab Syndr, 9, 42-45.

[29] Rochette L, Ghibu S and Muresan A. (2015). Alpha-lipoic acid molecular mechanisms and therapeutic potential in diabetes. Can J Physiol Pharmacol, 93, 1021-1027.

[30] HM El-sheikh, SM El-Haggar and TA Elbedewy. (2019). Comparative study to evaluate the effect of l-carnitine plus glimepiride versus glimepiride alone on insulin resistance in type 2 diabetic patients, Diabetes \& Metabolic Syndrome. Clinical Research \& Reviews, 13(1), 167-173.

[31] Boushel R, Gnaiger E, Schjerling P, Skovbro M, Kraunsoe R and Dela F. (2007). Patients with type 2 diabetes have normal mitochondrial function in skeletal muscle. Diabetologia, 50, 790-796.

[32] Patti ME, Butte AJ, Crunkhorn S, Cusi K, Berria R, Kashyap S, Miyazaki Y, Kohane I, Costello M and Saccone R. (2003). Coordinated reduction of genes of oxidative metabolism in humans with insulin resistance and diabetes: Potential role of PGC1 and NRF1. Proc. Natl. Acad. Sci, 100, 8466-8471. 
[33] Nishikawa T, Kukidome D, Sonoda K, Fujisawa K, Matsuhisa T, Motoshima H, Matsumura T and Araki E. (2007). Impact of mitochondrial ros production in the pathogenesis of insulin resistance. Diabetes Res. Clin. Pract, 77, 161-164.

[34] Kamz S and Sohal RS. (2004) Effect of age and caloric restriction on coenzyme Q and alpha-tocopherol levels in the rat. Exp.Gerotol, 39, 199-1205.

[35] Bour S, Carmana MC, Galinier A, Caspar-Bauguil S, Van Gaal L, Staels B, Penicaud L and Casteilla L. (2011). Coenzyme Q as an antiadipogenic factor. Antioxidants \& Redox Signaling, 14, 403-413.

[36] Blachnio-Zabielska A, Baranowski M, Zabielski P and Gorski J. (2010). Effect of high fat diet enriched with unsaturated and diet rich in saturated fatty acids on sphingolipid metabolism in rat skeletal muscle. J Cell Physiol, 225, 786-91.

[37] Hsu PP, Kang SA, Rameseder J, Zhang Y, Ottina KA, Lim D, Peterson TR, Choi Y, Gray NS, Yaffe MB, Marto JA and Sabatini DM. (2011). The mTOR-regulated Phosphoproteome Reveals a Mechanism of mTORC1-mediated Inhibition of Growth Factor. Signaling Science, 332, 1317-1322.

[38] McGlory C, Devries MC and Phillips SM. (1985). Skeletal muscle and resistance exercise training; the role of protein synthesis in recovery and remodeling. J Appl Physiol, 122, 541-548.

[39] Domingues-Faria C, Boirie Y and Walrand S. (2017). Vitamin D and Muscle Trophicity. Curr Opin Clin Nutr Metab Care, 20, 169-174.

[40] Kitakaze T, Harada N, Imag H and Yamaji R. (2015). $\beta$-Carotene Increases Muscle Mass and Hypertrophy in the Soleus Muscle in Mice. J Nutr Sci Vitaminol, 61, 481-7.

[41] Baracos V and RE AL. (1986). Influence of Calcium and it Divalent Cations on Protein Turnover in Rat Skeletal Muscle. Am J Physiol, 250, 702-710.

[42] Saltiel AR and Kahn CR. (2001). Insulin signalling and the regulation of glucose and lipid metabolism. Nature, 414, 799-806.

[43] Menon S, Dibble CC, Talbott G, Hoxhaj G, Valvezan AJ, Takahashi H, Cantley LC and Manning BD. (2014). Spatial control of the TSC complex integrates insulin and nutrient regulation of mTORC1 at the lysosome. Cell, 156, 771785.

[44] Liu YF, Wei JY, Shi MH, Jiang H and Zhou J. (2016). Glucocorticoid Induces Hepatic Steatosis by Inhibiting Activating Transcription Factor 3 (ATF3)/S100A9 Protein Signaling in Granulocytic Myeloid-derived Suppressor Cells. J Biol Chem, 291(41), 21771-21785.

[45] Shimizu N, Yoshikawa N, Ito N, Maruyama T, Suzuki Y, Takeda S, Nakae J, Tagata Y, Nishitani S, Takehana K, Sano M, Fukuda K, Suematsu M, Morimoto C and Tanaka H. (2011). Crosstalk between glucocorticoid receptor and nutritional sensor mTOR in skeletal muscle. Cell Metab, 13(2), 170-182.

\section{How to cite this article}

Emil M, Nailya I and Arailym A. (2020). Metabolic blocks and insulin resistance. World Journal of Advanced Research and Reviews, 6(2), 95-102. 